\title{
Trust and Reputation Modelling for Tourism Recommendations Supported by Crowdsourcing
}

\author{
Fátima Leal $^{1,3}$, Benedita Malheiro ${ }^{2,3}$, and Juan Carlos Burguillo ${ }^{1}$ \\ ${ }^{1}$ EET/UVigo - School of Telecommunication Engineering, University of Vigo, Spain \\ ${ }^{2}$ ISEP/IPP - School of Engineering, Polytechnic Institute of Porto, Portugal \\ 3 INESC TEC, Porto, Portugal \\ fatimaleal2@gmail.com, J.C.Burguillo@uvigo.es, mbm@isep.ipp.pt
}

\begin{abstract}
Tourism crowdsourcing platforms have a profound influence on the tourist behaviour particularly in terms of travel planning. Not only they hold the opinions shared by other tourists concerning tourism resources, but, with the help of recommendation engines, are the pillar of personalised resource recommendation. However, since prospective tourists are unaware of the trustworthiness or reputation of crowd publishers, they are in fact taking a leap of faith when then rely on the crowd wisdom. In this paper, we argue that modelling publisher Trust \& Reputation improves the quality of the tourism recommendations supported by crowdsourced information. Therefore, we present a tourism recommendation system which integrates: $(i)$ user profiling using the multi-criteria ratings; $(i i) k$-Nearest Neighbours $(k$-NN) prediction of the user ratings; (iii) Trust \& Reputation modelling; and (iv) incremental model update, i.e., providing near real-time recommendations. In terms of contributions, this paper provides two different Trust \& Reputation approaches: $(i)$ general reputation employing the pairwise trust values using all users; and ( $i i$ ) neighbour-based reputation employing the pairwise trust values of the common neighbours. The proposed method was experimented using crowdsourced datasets from Expedia and TripAdvisor platforms.
\end{abstract}

Keywords: Crowdsourcing, Trust \& Reputation, Rating Prediction, Tourism.

\section{Introduction}

Travel planning requires an attentive analysis by the tourist in order to choose the best tourism resources, i.e., transports, hotels, attractions, restaurants, etc. Information and Communication Technologies (ICT) promote smart tourism [8] and support the tourist, providing different platforms and applications to enhance the tourism experience [19, such as, social networks, mobile applications, crowdsourcing platforms, etc. In particular, the tourism crowdsourcing platforms have revolutionised the tourist behaviour [189]. These platforms (e.g., TripAdvisor, Expedia, Yelp, Booking.com, etc.), not only advertise tourism offers, but also promote the voluntary feedback sharing, which influences the behaviour 
of the other tourists. Therefore, tourism crowdsourced data analysis became a relevant research issue due to its impact in the tourist behaviour.

The crowdsourced information, which is voluntarily and freely shared, raises reliability questions. In this context, this paper explores the reliability of crowdsourced information using Trust \& Reputation (T\&R) modelling. While trustworthiness is a one-to-one relationship (intra-user or pairwise), reputation is a many-to-one relationship. First, we use multi-criteria tourism ratings to produce personalised tourism recommendations. Then, to ensure the reliability of both, information and users, we enrich the recommendation algorithm with T\&R information. Our proposed method includes: $(i)$ a profiling approach - Personalised Weighted Rating Average (PWRA) - based on multi-criteria ratings proposed by Leal et al. (2017) [15; ( $i i)$ Collaborative Filtering via $k$-NN; and (iii) two different T\&R models of crowdsourced data - general reputation and neighbourbased reputation. Since crowdsourced data corresponds to a stream of events, we propose an on-line recommendation approach via incremental updating, i.e., the model is updated whenever a new rating event occurs. In this work, user trustworthiness is based, according to the Leal et al. (2017) method [17, on the recommendations selected by the user and the reputation is modelled using the general and the neighbour-based approaches.

The main contributions of this paper are the $T \& R$ approaches designed for tourism recommendations supported by crowdsourced data: $(i)$ general user reputation based on the average user trustworthiness given by all acquaintances; and (ii) neighbour-based reputation built from the average user trustworthiness considering the set of common neighbours between two users. Our experiments with crowdsourced Expedia and TripAdvisor datasets show that both T\&R models improve significantly the $k$-NN rating prediction accuracy.

The rest of this paper is organised as follows. Section 2 reviews previous approaches of T\&R mechanisms for crowdsourced information. Section 3 describes the algorithms used in the methodology. Section 4 reports the results of the experiments and tests performed. Finally, Section 5 summarises and discusses the outcomes of this work.

\section{Related Work}

The crowdsourcing concept was introduced by Howe (2006) [10 to highlight the power of the crowd accomplishing different tasks, such as, funding, votes, texts, etc. Numerous systems have appeared on Web, including Wikipedia, Linux, Yahoo! Answers, Mechanical Turk-based systems, etc. [5].

In the case of tourism domain, platforms such as TripAdvisor, Booking, Expedia, etc. have influenced the tourist behaviour since they contain opinions of other tourists. The tourists create a digital footprint while organising and experiencing their trips, i.e., book and share their opinions in the form of ratings or textual reviews. Furthermore, a multitude of tourism applications which integrate profiling and recommendation algorithms support the tourist in the travel 
experience. These tourism systems use the digital footprints created by tourists to generate personalised suggestions.

The profiling mechanisms model the individuals using their digital footprint, employing information retrieval and data mining algorithms. In particular, the crowdsourcing platforms use multiple criteria ratings to classify the different aspects of a tourism resource (e.g., TripAdvisor uses for hotels the overall, location, rooms, cleanliness, value, etc.). The combination of this multi-criteria information is fundamental to refine the user profiles [1415. The resulting profiles are used by recommendation mechanisms to personalise suggestions. Regarding rating-based recommendation algorithms, collaborative filtering has long been used to create recommendations based on the ratings of users with similar preferences 7]13. Specifically, $k$-NN is the most popular memory-based collaborative filtering algorithm used to predict user preferences [1. Moreover, with $k$-NN, it is possible to know which users were responsible for the recommendations made.

Regarding the question of the reliability of crowdsourced information, we argue that T\&R modelling improves the quality of $k$-NN recommendations. Trust and Reputation are distinct, but intrinsically linked concepts, e.g., "I trust that hotel because it has good reputation". The literature contains different interpretations for trust and reputation. In this paper, we adopt the Jøsang et al. (2007) [12 interpretation for trust and reputation. Trust defines the reliability of users and resources based on direct experience, while Reputation is based on third party experiences, e.g., the crowd.

Scant research has been conducted regarding $T \& R$ in rating-based recommendation systems supported by crowdsourced tourism data. According to Richthammer et al. (2017) 20], reputation-enhanced recommender systems need further research. Richthammer et al. 20] present an updated survey regarding reputation coupled with recommendation systems. Particularly, in the tourism domain, Bedi et al. (2014) 2] propose a Multi-Agent Recommender System for e-Tourism (MARST) with item reputation modelling. The system has a dedicated agent for computing the reputation of tourism resources based on the number of users who rated the resource and the corresponding standard deviation. Bustos et al. (2009) [3] present the Social-Net Tourism Recommender System which integrates $\mathrm{T} \& \mathrm{R}$ modelling. The reputation is computed according to the number of edges of the user social network. Jøsang et al. (2013) [11] employ a discrete bayesian reputation algorithm using fuzzy membership functions.

Finally, taking into account the research addressing crowdsourced tourism data, we verify that the $T \& R$ modelling has not been explored yet. Therefore, our work contributes with a $T \& R$ user-based modelling for rating-based recommendation systems supported by crowdsourced tourism data, using incremental update and multi-criteria profiling.

\section{Proposed Method}

The proposed method explores the memory-based collaborative filtering to create a T\&R model. In this context, the method includes: (i) Profiling; (ii) T\&R 
Modelling ( $i i i$ ) On-line Rating Prediction; and (iv) Evaluation. The Profiling includes: $(i)$ the Personalised Weighted Rating Average (PWRA) proposed by Leal et al. (2017) [15]; (ii) the Trust modelling proposed by Leal et al. (2017) [17; and (iii) two different approaches for reputation modelling - General Reputation and Neighbour-based Reputation - which are the contributions of this paper. For the On-line Rating Prediction we employ the $k$-NN algorithm and use Pearson correlation to identify the nearest neighbours. The Evaluation is performed using Root Mean Squared Error (RMSE), Target Recall (TRecall) and Recall. Our method applies incremental updating (data streaming), i.e., the model is updated every time a new event occurs.

\subsection{Profiling}

Personalised Weighted Rating Average (PWRA) explores the multicriteria ratings in order to refine the user profile. Equation 1 combines the multiple criteria ratings, i.e., cleanliness, room service, staff, etc., into a single rating $\left(r_{u, i}\right)$ using a personalised weighted average of the non-null criterion ratings. Therefore, the $r_{u, i, c}$ is the non-null rating of the criterion $c$ given by user $u$ to item $i$. The $n_{c}$ presents the number of times which the user $u$ has rated the criterion $c$. The $n_{u, c}$ is the number of non-null ratings of criterion $c$ given by user $u$, and, finally, $n$ is the total number of non-null multi-criteria ratings given by user $u$.

$$
r_{u, i}=\frac{\sum_{c=1}^{n} n_{c} r_{u, i, c}}{\sum_{c=1}^{n} n_{u, c}}
$$

\subsection{Trust \& Reputation Modelling}

Tourism collaborative recommendation engines rely on tourist feedback to make recommendations to prospective tourists. Facing this scenario, we propose a T\&R model to build higher-quality recommendations. The model involves: (i) Similarity Measuring; (ii) Trustworthiness; and (iii) Final Reputation. The model is updated upon each user event. The similarity measuring relies on Pearson Correlation metrics to detect the nearest neighbours. Additionally, this metric provides the correlation values which are used in the rating predictions. The trustworthiness qualifies the relationship between users, taking into account the number of times which the current user selects one of the top 10 recommendations provided by his/her neighbours. The Final Reputation combines the trustworthiness values using two different approaches: $(i)$ general reputation; and (ii) neighbour-based reputation.

Similarity Measuring relies on Pearson Correlation Coefficient to measure the correlation (proximity) between two users 21. Equation 2 calculates the Pearson Correlation $\left(P C_{u, k}\right)$ between user $u$ and user $k$. The parameter $r_{u, i}$ is the rating given by user $u$ to the item $i ; \bar{r}_{u}$ and $\bar{r}_{k}$ are the average of the 
co-rated items given by user $u$ and $k$, respectively; $r_{k, i}$ is the rating given by neighbour $k$ to the item $i$; and $m$ is the total number of items [6].

$$
P C_{u, k}=\frac{\sum_{i=1}^{m}\left[\left(r_{u, i}-\bar{r}_{u}\right)\left(r_{k, i}-\bar{r}_{k}\right)\right]}{\sqrt{\sum_{i=1}^{m}\left(r_{u, i}-\bar{r}_{u}\right)^{2} \sum_{i=1}^{m}\left(r_{k, i}-\bar{r}_{k}\right)^{2}}}
$$

Trustworthiness quantifies the relationship between a user and his/her neighbours. It takes into account the resulting recommendations and subsequent user selections. Therefore, if a given user $u$ selects multiple recommendations due to a neighbour $k$, the trustworthiness between of $u$ in $k$ increases. The trustworthiness is updated every time the user selects and rates a recommendation. Equation 3 computes the trustworthiness $T_{u, k}$ between user $u$ and $k$ where $n_{u, k}$ represents the number of items actually recommended to $u$ due to $k$ and $N_{u, k}$ the number of times $k$ was chosen as a neighbour of $u$.

$$
T_{u, k}=\frac{n_{u, k}}{N_{u, k}}
$$

Reputation quantifies the quality of all contributions of a given user. However, while the trustworthiness defines the reliability of users based on direct experience, reputation is based on the crowd perspective. Therefore, we propose two different approaches for the user reputation modelling: $(i)$ a general user reputation; and (ii) neighbour-based reputation, i.e., the user reputation according to its neighbours.

- General Reputation quantifies the reputation of a user using the pairwise trustworthiness between the user and each one of his/her acquaintances. Equation 4 displays the general reputation $R_{u}$ of a user $u$ in the system. It corresponds to the average trustworthiness $T_{u, k}$ of user $u$ according to the system users $U$.

$$
R_{u}=\frac{\sum_{k=1}^{U} T_{u, k}}{U}
$$

- Neighbour-based Reputation quantifies the reputation of a user using the pairwise trustworthiness of the neighbours shared between the user and each one of his/her acquaintances. Equation 5 displays the neighbour-based reputation $R_{u, k}$ of the user $u$ according to user $k$ where $t$ represents the common neighbours between $u$ and $k ; T_{u, t}$ is the pairwise trustworthiness between the user $u$ and $t$; and $n$ is the number of the total shared neighbours between $u$ and $k$.

$$
R_{u, k}=\frac{\sum_{t=1}^{n} T_{u, t}}{n}
$$

Equation 6 computes the final user reputation $R_{u_{T}}$ using the common neighbours of all users in the system, where $R_{u, k}$ is the neighbour-based reputation of the user $u$ based on user $k$, and $U$ is the total number of users in the system.

$$
R_{u_{T}}=\frac{\sum_{t=1}^{U} R_{u, t}}{U}
$$




\subsection{On-line Rating Prediction}

The rating prediction relies on the $k$-NN algorithm. The method predicts and updates the model in near real time for each incoming rating event. In order to identify the nearest neighbours, we employ Pearson Correlation. The Pearson algorithm determines the correlation among users to detect the nearest neighbours. Therefore, the $k$ users with higher Pearson Correlation with the active user are his/her nearest neighbours.

$k$-Nearest Neighbours is widely used in collaborative filtering. Our proposed method employs $k$-NN to determine the $k$ user neighbours (neighbourhood) and, thus, generate recommendations for the active user $u$. The generated rating predictions are used to identify the most promising of the following four profiling approaches:

Default approach employs the Equation 1 to compute the $r_{u, i}$ using the multicriteria ratings. Then, the prediction of the rating given by user $u$ to item $i$, $\hat{r}_{u, i}$, is determined with the help of Equation 7, where $P C_{u, k}$ is the correlation between the user $u$ and user $k, \bar{r}_{u}$ and $\bar{r}_{k}$ are the average of the co-rated items given by user $u$ and $k$, respectively, $r_{k, i}$ is the rating given by neighbour $k$ to the item $i$, and $n$ the number total of neighbours.

$$
\hat{r}_{u, i}=\bar{r}_{u}+\frac{\sum_{k=1}^{n}\left[\left(r_{k, i}-\bar{r}_{k}\right) * P C_{u, k}\right]}{\sum_{k=1}^{n} P C_{u, k}}
$$

Trust based approach, which was presented by Leal et al. (2017) [17, combines PWRA and trust modelling as described in Equation 3 . This approach computes the number of times a user $k$ was identified as a neighbour of a user $u$ and the number of the items selected by $u$ due to neighbour $k$. Equation 8 displays the rating prediction $\hat{r}_{T_{u, i}}$ of item $i$ for a user $u$ employing the trustworthiness $T_{u, k}$ of the $n$ neighbours of user $u$.

$$
\hat{r}_{T_{u, i}}=\hat{r}_{u, i} * \frac{\sum_{k=1}^{n} T_{u, k}}{n}
$$

General Reputation approach applies the general reputation $R_{u}$ of user $u$ in the system, obtained through Equation 4 to the predicted rating $\hat{r}_{u, i}$, determined by Equation 7 . Equation 9 predicts the rating of item $i$ for user $u$ according to the general reputation $\hat{r}_{G R_{u, i}}$.

$$
\hat{r}_{G R_{u, i}}=\hat{r}_{u, i} * R_{u}
$$

Neighbour-based Reputation approach applies the neighbour based reputation $\hat{r}_{N R_{u, i}}$ of Equation 6 to the predicted rating $\hat{r}_{u, i}$, determined by Equation 7. Therefore, we use Equation 10 to predict the $\hat{r}_{N R_{u, i}}$ of item $i$ for user $u$ using the neighbour-based reputation $R_{u_{T}}$ as filter to improve the final recommendations.

$$
\hat{r}_{N R_{u, i}}=\hat{r}_{u, i} * R_{u_{T}}
$$




\subsection{Evaluation Metrics}

The evaluation is performed by calculating the RMSE, Recall and TRecall. The RMSE measures the error between the predicted rating and the real user rating. We calculate incrementally the overall RMSE error, i.e., whenever a new event occurs, according to the Takács et al. (2009) approach [22. The Recall metric proposed by Cremonesi et al. (2010) 4 and the Target Recall presented by Veloso et al. (2017) 23] evaluate the recommendations accuracy. To calculate the Recall, first, we predict the ratings of all items non rated by the user, including the newly rated item. Then, we do a random selection of 1000 non rated items, add the newly rated item, and sort these 1001 events in descending order. Finally, if the new event belongs to the list of the top $N$ recommendations, it counts as a hit. In the case of TRecall, there is a hit whenever the predicted rating lays within the $\pm \frac{N}{2}$ predictions centred on the real rating. Therefore, to evaluate our on-line method we use RMSE, the Recall@N, and the TRecall@N, with $N=10$.

\section{Experiments and Results}

The experiments involved the four PWRA based profiling approaches: $(i)$ Default; (ii) Trust; (iii) General Reputation; and (iv) Neighbour-based Reputation. In each case, we predict the user ratings using the $k$-NN algorithm. Finally, we evaluate each method considering its prediction and recommendation accuracy. Our recommendation engine is implemented in Java, runs on an OpenStack cloud instance with $16 \mathrm{GiB}$ RAM, $8 \mathrm{CPU}$ and $160 \mathrm{GiB}$ of hard-disk space. The proposed method was tested using two different datasets - HotelExpedia dataset and TripAdvisor. The data was ordered temporally (i.e., data streams) and, then, partitioned. The initial model uses the $20 \%$ of the dataset, and the remaining $80 \%$ of the dataset is used for incremental updates. The model is updated whenever a user introduces a new rating.

\subsection{Data Sets}

We conducted several experiments with the Expedia and TripAdvisor data in order to validate and evaluate our proposed method.

HotelExpedia dataset was collected by Leal et al. (2017) [16. It is composed by 6276 hotels, 1090 identified users and 214342 reviewers from 11 different locations. Each user classified at least 20 hotels, and each hotel has a minimum of 10 ratings. The dataset holds the following multi-criteria ratings: the overall, cleanliness, service 83 staff, hotel condition and room comfort.

TripAdvisor dataset was proposed by Wang et al. (2010) 24]. It is composed by 9114 hotels, 7452 users and 127517 hotel reviews. Each user classified at least 20 hotels, and each hotel has a minimum of 10 ratings. The dataset has as multi-criteria ratings: overall, value, rooms, location, cleanliness, service, and sleep quality. 


\subsection{Recommendation Results}

The T\&R modelling aims to refine the final recommendations. Table 1 compares the RMSE, Recall and TRecall with: (i) Default (Equation 7); (ii) Trust (Equation 8); (iii) General Reputation (Equation 9); and (iii) Neighbour-based Reputation (Equation 10). Lower error values and higher classification values indicate higher prediction accuracy. The number of neighbours was applied according to Leal et al. 17] study for HotelExpedia and TripAdvisor datasets. The Default profiling, which corresponds of the just to PWRA profiling, is the base approach.

Table 1. Comparison of Recommendation Results

\begin{tabular}{|c|c|c|c|c|c|}
\hline & Profiling & Neighbours & Recall@10 & TRecall@10 & RMSE \\
\hline \multirow{4}{*}{ Hotel Expedia } & Default: $\hat{r}_{u, i}$ & 20 & 0.304 & 0.198 & 0.129 \\
\hline & Trust: $\hat{r}_{T} u, i$ & 20 & 0.639 & 0.215 & 0.124 \\
\hline & General Reputation: $\hat{r}_{G_{u, i}}$ & 20 & 0.679 & 0.235 & 0.119 \\
\hline & Neighbour-based Reputation: $\hat{r}_{N R_{u, i}}$ & 20 & 0.719 & 0.247 & 0.117 \\
\hline \multirow{4}{*}{ Trip Advisor } & Default: $\hat{r}_{u, i}$ & 200 & 0.489 & 0.513 & 0.152 \\
\hline & Trust: $\hat{r_{T}, i}$ & 200 & 0.823 & 0.621 & 0.132 \\
\hline & Reputation: $\hat{r}_{G_{u, i}}$ & 200 & 0.837 & 0.630 & 0.128 \\
\hline & Neighbour-based Reputation: $\hat{r}_{N R_{u, i}}$ & 200 & 0.859 & 0.639 & 0.125 \\
\hline
\end{tabular}

We can verify that the most promising results occurred with neighbour-based reputation. In the case of the HotelExpedia dataset, when compared with the base algorithm, the RMSE decreases $(-9 \%)$ and the Recal@10 (+137\%) and TRecall@10 (+25\%) increase. In the case of the TripAdvisor dataset, when compared with the base algorithm, the RMSE decreases (-18\%). The Recall@10 $(+76 \%)$ and TRecall@10 (+25\%) increase.

\section{Conclusions}

Prospective tourists rely increasingly on crowdsourcing platforms to plan their trips, reinforcing the influence of crowdsourced information in the tourist behaviour. In this context, past tourists leave behind digital footprints composed of multiple criteria information such as ratings, textual reviews, photos, etc., which are used to recommend tourism resources. To improve the quality of these recommendations, we assess crowdsourced information using T\&R mechanisms.

We explore the T\&R modelling of tourism crowdsourced information in order to create on-line collaborative recommendations. Our proposed method relies on data streams and includes: (i) PWRA multi-criteria Profiling; (ii) On-line Rating Prediction; ( $i i i)$ T\&R modelling; and (iv) Evaluation. The Profiling module combines the multi-criteria ratings to create the profiles using the PWRA approach. For rating prediction, we use a collaborative filtering employing the $k$-NN algorithm to predict the ratings, and the neighbourhood is computed using the Pearson correlation as the similarity measure. Additionally, we integrate 
a T\&R modelling to refine users' profiles, and, consequently, the final recommendations. First, the trustworthiness between users is computed taking into account the number of items actually recommended to the user based on the neighbour, and the number of times that a user was chosen as a neighbour of the current one. Then, we explore two different reputation approaches: $(i)$ a general reputation, which relies on a standard average of the pairwise trust values taking into account all users of the system; and (ii) neighbour-based reputation, which relies on a standard average of the pairwise trust values taking into account the common neighbours between users.

The proposed method was tested and evaluated with Expedia and TripAdvisor datasets, using RMSE, Recall@10, and TRecall@10 as evaluation metrics and employing incremental updating. In the experiments, we compared four different approaches: ( $i$ ) Default; (ii) Trust; (iii) General Reputation; and (iv) Neighbourbased reputation. The most promising results occurred with neighbour-based reputation for both datasets.

To sum up, this paper proposes a T\&R modelling approach for recommendation systems supported by crowdsourced ratings. As future work, we intend to explore the T\&R modelling for model-based recommendation systems.

\section{Acknowledgements}

This work was partially financed by: $(i)$ the European Regional Development Fund (ERDF) through the Operational Programme for Competitiveness and Internationalisation - COMPETE 2020 Programme - within project «POCI-010145-FEDER-006961», and by National Funds through Fundação para a Ciência e a Tecnologia (FCT) - the Portuguese Foundation for Science and Technology - as part of project UID/EEA/50014/2013; and (ii) ICT COST Action IC1406 High-Performance Modelling and Simulation for Big Data Applications (cHiPSet).

\section{References}

1. C. C. Aggarwal. Neighborhood-based collaborative filtering. In Recommender Systems, pages 29-70. Springer, 2016.

2. P. Bedi, S. K. Agarwal, V. Jindal, et al. Marst: Multi-agent recommender system for e-tourism using reputation based collaborative filtering. In International Workshop on Databases in Networked Information Systems, pages 189-201. Springer, 2014.

3. F. Bustos, J. López, V. Julián, and M. Rebollo. Strs: Social network based recommender system for tourism enhanced with trust. In International Symposium on Distributed Computing and Artificial Intelligence 2008 (DCAI 2008), pages 71-79. Springer, 2009.

4. P. Cremonesi, Y. Koren, and R. Turrin. Performance of recommender algorithms on top-n recommendation tasks. In RecSys '10, pages 39-46, Barcelona, Sept. 2010. ACM.

5. A. Doan, R. Ramakrishnan, and A. Y. Halevy. Crowdsourcing systems on the world-wide web. Communications of the ACM, 54(4):86-96, 2011. 
6. M. D. Ekstrand, J. T. Riedl, J. A. Konstan, et al. Collaborative filtering recommender systems. Foundations and Trends in Human-Computer Interaction, $4(2): 81-173,2011$.

7. D. Goldberg, D. Nichols, B. M. Oki, and D. Terry. Using collaborative filtering to weave an information tapestry. Communications of the ACM, 35(12):61-70, 1992.

8. U. Gretzel, M. Sigala, Z. Xiang, and C. Koo. Smart tourism: foundations and developments. Electronic Markets, 25(3):179-188, 2015.

9. I. Gula. Crowdsourcing in the tourism industry - using the example ofideas competitions in tourism destinations. In ISCONTOUR 2013: Proceedings of the International Student Conference in Tourism Research, page 147. BoD-Books on Demand, 2013.

10. J. Howe. The rise of crowdsourcing. Wired magazine, 14(6):1-4, 2006.

11. A. Jøsang, G. Guo, M. S. Pini, F. Santini, and Y. Xu. Combining recommender and reputation systems to produce better online advice. In International Conference on Modeling Decisions for Artificial Intelligence, pages 126-138. Springer, 2013.

12. A. Jøsang, R. Ismail, and C. Boyd. A survey of trust and reputation systems for online service provision. Decision support systems, 43(2):618-644, 2007.

13. Y. Koren and R. Bell. Advances in collaborative filtering. In F. Ricci, L. Rokach, and B. Shapira, editors, Recommender Systems Handbook, chapter 3, pages 77-118. Springer US, Boston, MA, 2015.

14. K. Lakiotaki, P. Delias, V. Sakkalis, and N. F. Matsatsinis. User profiling based on multi-criteria analysis: the role of utility functions. Operational Research, 9(1):316, 2009.

15. F. Leal, H. González-Vélez, B. Malheiro, and J. C. Burguillo. Profiling and rating prediction from multi-criteria crowd-sourced hotel rating. In Proceedings of the 31th European Conference on Modelling and Simulation, ECMS 2017, pages 576-582. ECMS, 2017.

16. F. Leal, B. Malheiro, and J. C. Burguillo. Prediction and Analysis of Hotel Ratings from Crowd-Sourced Data, pages 493-502. Springer International Publishing, Cham, 2017.

17. F. Leal, B. Malheiro, H. González-Vélez, and J. C. Burguillo. Trust-based modelling of multi-criteria crowdsourced data. Data Science and Engineering, Sep 2017.

18. B. Neuhofer. Innovation through co-creation: Towards an understanding of technology-facilitated co-creation processes in tourism. In Open Tourism, pages 17-33. Springer, 2016.

19. B. Neuhofer, D. Buhalis, and A. Ladkin. A typology of technology-enhanced tourism experiences. International Journal of Tourism Research, 16(4):340-350, 2014.

20. C. Richthammer, M. Weber, and G. Pernul. Reputation-enhanced recommender systems. In IFIP International Conference on Trust Management, pages 163-179. Springer, 2017.

21. P. Sedgwick. Pearson's correlation coefficient. British Medical Journal, 345:e4483, 2012.

22. G. Takács, I. Pilászy, B. Németh, and D. Tikk. Scalable Collaborative Filtering Approaches for Large Recommender Systems. The Journal of Machine Learning Research, 10:623-656, June 2009.

23. B. Veloso, B. Malheiro, J. C. Burguillo, and J. Foss. Personalised fading for stream data. In SAC 2017, pages 870-872, Marrakech, Apr. 2017. ACM.

24. H. Wang, Y. Lu, and C. Zhai. Latent aspect rating analysis on review text data: a rating regression approach. In KDD '10, pages 783-792, Washington, July 2010. ACM. 